\title{
Fabrication of Spin-Transfer Nano-Oscillator by Colloidal Lithography
}

\author{
Bin Fang, ${ }^{1}$ Jiafeng Feng, ${ }^{2}$ Hongxiang Wei, ${ }^{2}$ Xiufeng Han, ${ }^{2}$ \\ Baoshun Zhang, ${ }^{1}$ and Zhongming Zeng ${ }^{1}$ \\ ${ }^{1}$ Key Laboratory of Nanodevices and Applications, Suzhou Institute of Nano-Tech and Nano-Bionics, Chinese Academy of Sciences, \\ Ruoshui Road 398, Suzhou 215123, China \\ ${ }^{2}$ Beijing National Laboratory for Condensed Matter Physics, Institute of Physics, Chinese Academy of Sciences, Beijing 100190, China \\ Correspondence should be addressed to Hongxiang Wei; hxwei@iphy.ac.cn and Zhongming Zeng; zmzeng2012@sinano.ac.cn
}

Received 18 September 2015; Revised 23 November 2015; Accepted 24 November 2015

Academic Editor: Stefano Bellucci

Copyright (C) 2015 Bin Fang et al. This is an open access article distributed under the Creative Commons Attribution License, which permits unrestricted use, distribution, and reproduction in any medium, provided the original work is properly cited.

\begin{abstract}
We fabricate nanoscale spin-transfer oscillators (STOs) by utilizing colloidal nanoparticles as a lithographic mask. By this approach, high quality STO devices can be fabricated, and as an example the fabricated STO devices using MgO magnetic tunnel junction as the basic cell exhibit current-induced microwave emission with a large frequency tunability of $0.22 \mathrm{GHz} / \mathrm{mA}$. Compared to the conventional approaches that involve a step of defining nanoscale elements by means of electron beam lithography, which is not readily available for many groups, our strategy for STO fabrication does not require the sophisticated equipment $(\sim$ million dollars per unit) and expensive lithography resist, while being cost-effective and easy to use in laboratory level. This will accelerate efforts to implement STO into on-chip integrated high-radio frequency applications.
\end{abstract}

\section{Introduction}

Spin-transfer oscillators (STOs) have recently aroused tremendous and continuous research interest due to their nanoscale size, frequency tunability, broad temperature operation range, and Si technology compatibility [1-3]. STO devices utilize spin-transfer torque (STT) generated by a spinpolarized current traversing a nanoscale magnetic multilayer $[4,5]$, such as spin valve and magnetic tunnel junctions (MTJs) that consist of a free layer and reference layer separated by a nonmagnetic metal or insulator layer. STT can excite steady processions of the free layer magnetization, leading to emission of a microwave signal [6-10]. In 2003, the microwave signal generated by STT was firstly demonstrated in $\mathrm{Co} / \mathrm{Cu} / \mathrm{Co}$ spin valve nanopillars [6]. However, the output power is far low (on the level of $\mathrm{pW}$ ). Since that, many efforts, including optimization of device geometry and material structure, and the control of applied magnetic field directions, have been made to improve STO performances, that is, enhancing output power, narrowing spectral linewidth, or removing the need of the external magnetic field [11-17].
For most experiments performed to date, in order to fabricate nanoscale STO devices, the high-performance electron beam lithography (EBL) system was required [6]. However, the expensive EBL system is not readily available to many groups. Furthermore, EBL process is time consuming and limited to small scales. These issues of EBL may partly hinder STO research.

It is well known that the colloidal nanospheres are widely used to fabricate nanostructures with a wide range of sizes [18]. Recently, Wang et al. [19] demonstrated that magnetic tunnel junction device can be fabricated by using nanospheres ( $\sim 400 \mathrm{~nm}$ in diameter) as the lithographic mask. The fabricated MTJs showed an obvious switching for magnetic random access memory. They also mentioned that it is challenging to produce small nanopillars $(\sim 100 \mathrm{~nm})$ due to difficulties in the lift-off of nanospheres after $\mathrm{SiO}_{2}$ deposition. However, for spin-torque nano-oscillators, in order to observe microwave signal, it is generally required to fabricate the smaller nanopillars ( $\sim 100 \mathrm{~nm}$ in lateral) for enabling large d.c. current density to excite magnetization. In this study, we used the nanospheres with a diameter of $160 \mathrm{~nm}$ as a 

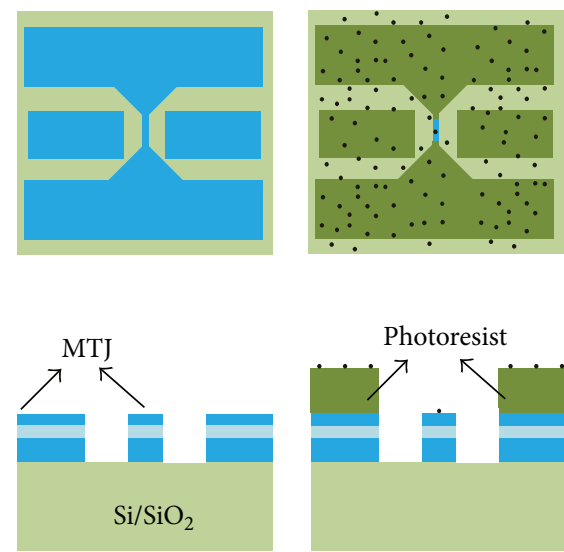

(a)

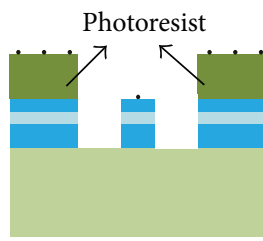

(b)
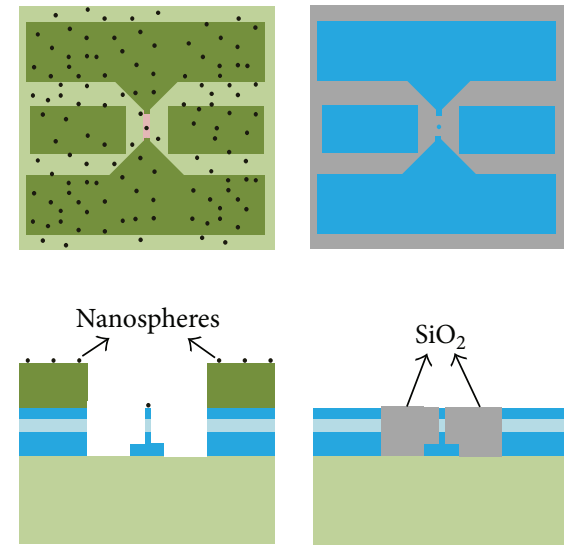

(c)

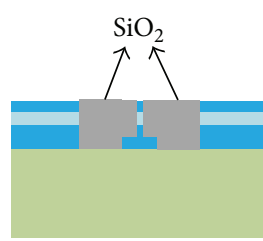

(d)
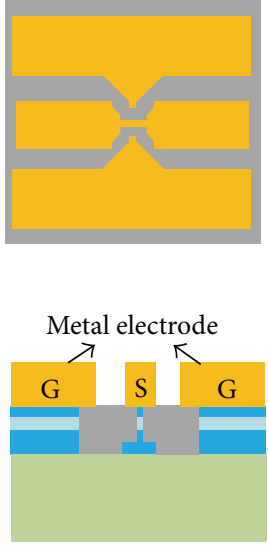

(e)

FIgURE 1: Nanopillar fabrication process. (a) Patterning contact pads and the mesa by optical lithography and Argon ion etching. (b) Protecting the contacts by photoresist and coating the polystyrene nanospheres. (c) Argon ion etching. (d) Depositing $\mathrm{SiO}_{2}$ insulating layer and removing the nanospheres and photoresist. (e) Depositing the contacts through photolithography and e-beam evaporation.

mask and have fabricated successfully smaller nanopillars by optimizing the lift-off process. The STOs fabricated by this approach exhibited a steady-state magnetization procession, which can be controlled by d.c. current or magnetic field. Our findings show that the strategy of combination of colloidal lithography and optical lithography provides an alternative, simple, and cost-effective method for STO research and applications.

\section{Experimental Details}

The fabrication process begins a MTJ stack of bottom contact $/ \mathrm{PtMn} 15 / \mathrm{Co}_{70} \mathrm{Fe}_{30}$ 2.3/Ru 0.85/ $\mathrm{Co}_{40} \mathrm{Fe}_{40} \mathrm{~B}_{20} 2.4 / \mathrm{MgO}$ $0.8 / \mathrm{Co}_{20} \mathrm{Fe}_{60} \mathrm{~B}_{20}$ 1.8/top contact (the thicknesses are in nanometers), sputtered onto the thermally oxidized $\mathrm{Si}$ wafer by sputter system. The synthetic antiferromagnetic $\mathrm{Co}_{70} \mathrm{Fe}_{30} /$ $\mathrm{Ru} / \mathrm{Co}_{40} \mathrm{Fe}_{40} \mathrm{~B}_{20}$ reference layer, exchange biased by a PtMn layer, is designed to have an in-plane easy axis. The $\mathrm{Co}_{20} \mathrm{Fe}_{60} \mathrm{~B}_{20}$ free layer has also an in-plane easy axis at zero external magnetic field, but with strong perpendicular magnetic anisotropy at the interface of the CoFeB layer with the $\mathrm{MgO}$ tunnel barrier [11]. This perpendicular anisotropy can partially cancel the demagnetization field in the free layer [13]; thus this structure in essence enables to excite large-amplitude magnetization precession under a small bias current.

Standard photolithography and Ar ion milling were used to pattern the contact pads and the $6 \times 50 \mu \mathrm{m}^{2}$ mesas as shown in Figure 1(a). Then the second photolithography step was introduced to open a window that allows the polystyrene nanospheres to adhere to the top of the mesas. The photoresist was also used to protect the contact pads from the nanospheres and $\mathrm{SiO}_{2}$ insulator deposition. The next step is a key part of the fabrication process that defines the MTJ nanopillars by colloidal lithography. The nanospheres with a diameter of $160 \mathrm{~nm}$ (see Figure 2(a)) were suspended in a deionized- (DI-) based solvent by ultrasound 3 minutes in order to improve dispersity. Before coating spheres, the wafer was coated with hexamethyldisilazane (HMDS) that strengthens adhesion between wafer and nanospheres; the spheres were spin-coated on the surface to act as etching mask for subsequent etching (Figure 1(b)). After that the Argon ion milling was used to etch the materials without protection by photoresist and nanospheres (Figures 1(c) and 2(b)). This was followed by depositing a $50 \mathrm{~nm} \mathrm{SiO}_{2}$ insulator layer by Inductively Coupled Plasma CVD (ICPCVD) system at low temperature $\left(\sim 75^{\circ} \mathrm{C}\right)$. Next, the wafer was dipped in dichloromethane and resist-remover to remove the spheres as well as the photoresist (Figures 1(d) and 2(c)). The fabrication of devices is completed by contacting the nanopillars and the two ground contacts to form a Ti $(10 \mathrm{~nm}) / \mathrm{Au}$ $(100 \mathrm{~nm})$ ground-signal-ground (GSG) waveguide, deposited using electron beam evaporation and patterned using optical lithography and lift-off (Figure 1(e)). The final device is shown in Figure 2(d).

The measurement setup of STT-induced microwave oscillation is similar to that described in the previous work [13]. In the experiments, the d.c. current was applied to the device through a bias Tee, where we define the positive current $I_{\text {d.c. }}$ as electrons flowing from the polarizer to the free layer. The microwave voltages generated by STT were recorded by a spectrum analyzer with an amplification of $40 \mathrm{~dB}$ and a baseline taken at $I_{\text {d.c. }}=0 \mathrm{~mA}$ was subtracted from the measured spectral data. All measurements were carried out at room temperature.

\section{Results and Discussion}

Before microwave signal generation study, we measured the transport properties under an external magnetic field for understanding the magnetization states. Figure 3(a) presents a magnetoresistance loop corresponding to the switching of the free layer between the low-resistance $\left(R_{\mathrm{p}}\right)$ parallel state and the high-resistance $\left(R_{\mathrm{AP}}\right)$ antiparallel alignment with a 


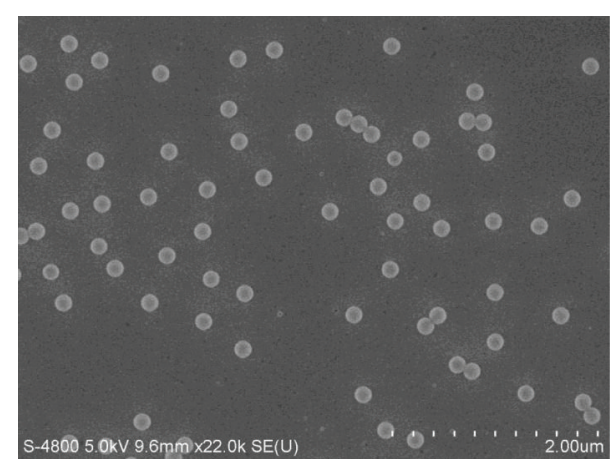

(a)

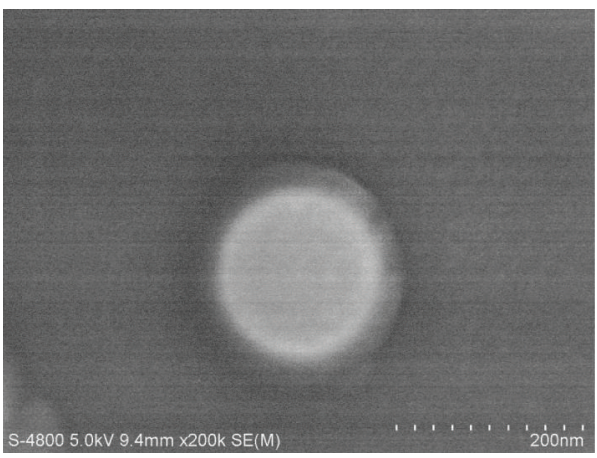

(c)

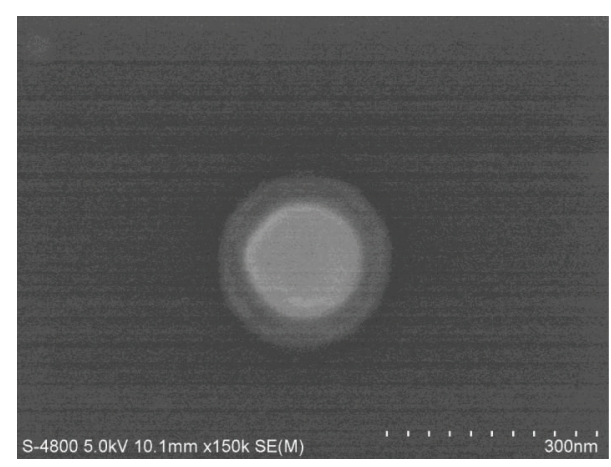

(b)

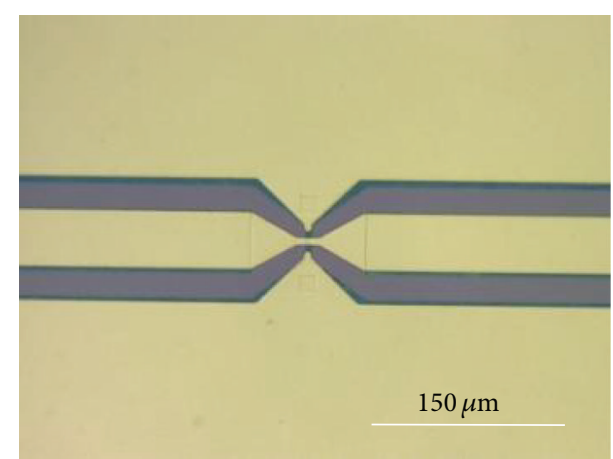

(d)

FIGURE 2: (a) Scanning electron microscopy (SEM) image of polystyrene nanospheres on wafer. (b) SEM image for MTJ nanopillar with nanosphere after Argon ion etching, (c) SEM image of nanopillar after removing nanosphere and $\mathrm{SiO}_{2}$, and (d) optical image of the final STO device.

bias current $I_{\text {d.c. }}=10 \mu \mathrm{A}$. The TMR ratio, defined as TMR = $100 \times\left(R_{\mathrm{AP}}-R_{\mathrm{P}}\right) / R_{\mathrm{P}}$, and the resistance-area (RA) product in the parallel state were $93 \%$ and $1.1 \Omega \mu \mathrm{m}^{2}$, respectively. The low RA allows the sufficient d.c. current to excite the microwave oscillations [11]. A small loop shift was observed, which is due to the ferromagnetic coupling through barrier roughness and antiferromagnetic dipolar interaction between layers [9].

All devices exhibited microwave signal generation when driven with sufficiently large d.c. current, even without the application of external magnetic field. For the device presented above, we observed obvious high-frequency signal for currents above $I_{\text {d.c. }}=0.3 \mathrm{~mA}$, which corresponds to a critical threshold current density $\left(J_{\text {th }}\right)$ of $1.5 \mathrm{MA} / \mathrm{cm}^{2}$ for microwave generation. Note that the $J_{\mathrm{c}}$ value can be reduced if the thinner $\mathrm{CoFeB}$ free layer is used since the interfacial perpendicular anisotropy between the $\mathrm{CoFeB}$ electrodes and the tunnel barrier of the MTJ increases with decreasing the thickness of the CoFeB free layer [20]. For currents above $0.3 \mathrm{~mA}$, two peaks with Lorentzian line shape were observed for each current as shown in Figure 3(b). The lowerfrequency peak may be attributed to a centre precession mode while the higher-frequency peak probably arises from a mode with large amplitude at the sides of the nanopillar. Quantitative data are gathered in Figure 4, where we report the frequencies, powers, and linewidths of the centre precession mode as a function of applied current. First, a blueshift in the oscillation frequencies with increasing the d.c. current is observed. For $0.5 \mathrm{~mA} \leq I_{\text {d.c. }} \leq 1.5 \mathrm{~mA}$, the frequencies increase rapidly, resulting in a current modulation capability of $0.22 \mathrm{GHz} / \mathrm{mA}$. Further increasing current, the frequencies exhibit a slight increase. The similar trend has been also observed in other systems [21], which suggests that the steady-state excitation corresponds to an out-ofplane precession. Second, the integrated power of the centre mode increases gradually with increasing the d.c. current and reaches $5 \mathrm{nW}$ at $I_{\text {d.c. }}=4 \mathrm{~mA}$.

Figure 4(c) shows the linewidth of the centre mode as a function of the d.c. current. We observed a relatively broad linewidth of $0.74 \mathrm{GHz}$ just above threshold at low currents. For $0.5 \mathrm{~mA} \leq I_{\text {d.c. }} \leq 1.5 \mathrm{~mA}$, the linewidth narrows quasilinearly as the current is increased, while the linewidth narrows slightly above $1.5 \mathrm{~mA}$. This trend can be understood as follows. According to the nonlinear theories [2, 22], the linewidth $\Delta f$ in the above threshold regime depends not only on the frequency nonlinearity $N=\mathrm{d} f / \mathrm{d} I_{\text {d.c. }}$, but also on the oscillation energy that is proportional to the output power $P$; that is, $\Delta f \propto N / P$. It can be seen from Figure 4(a) that the frequency shift decreases, suggesting the frequency nonlinearity $N$ decreases with increasing the currents. Meanwhile, the output power has an ascending dependence on the currents as shown in Figure 4(b). As a result, it is explicit that the current dependence of $\Delta f$ observed in this study is reasonable. 


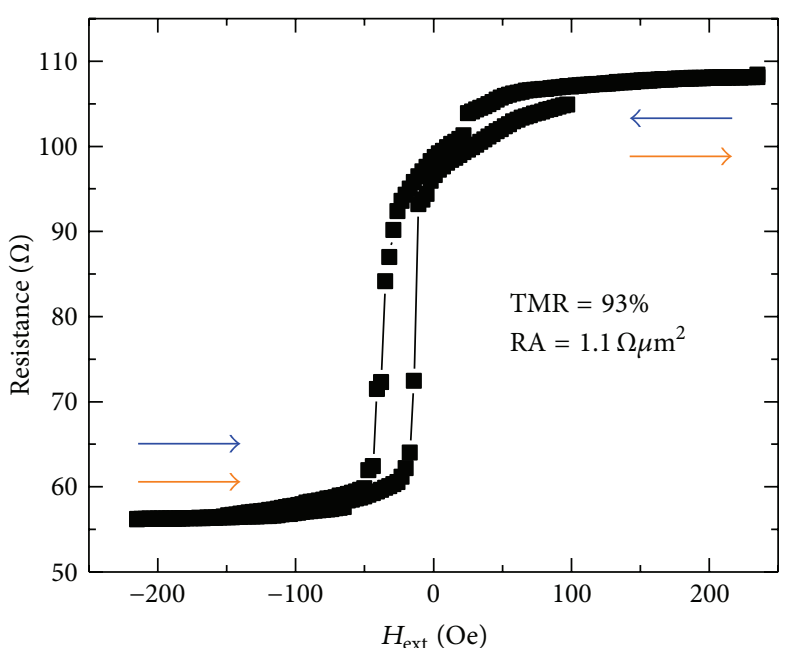

(a)

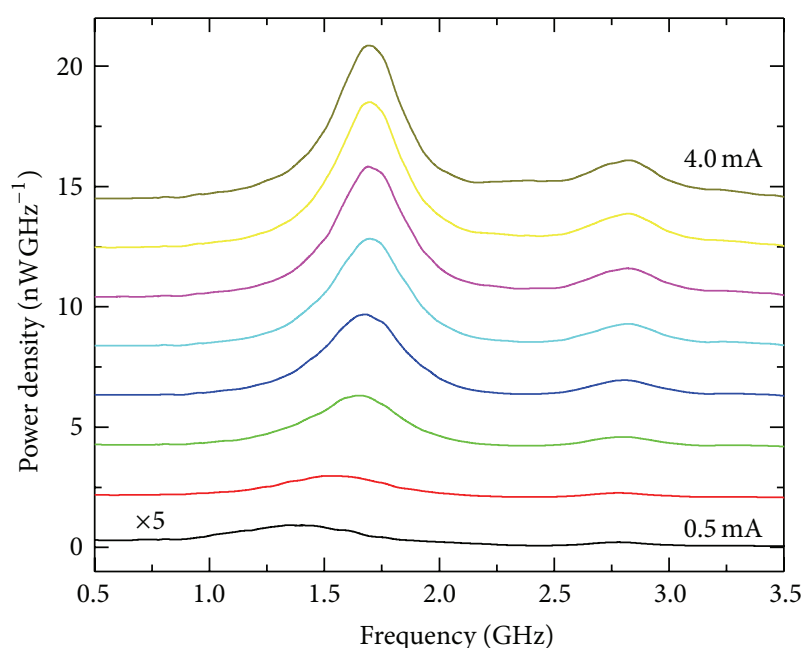

(b)

FIgURE 3: (a) The device resistance as a function of applied magnetic field alone in-plane easy axis under bias current of $10 \mathrm{uA}$. The orange/blue arrow represents the magnetization direction of the reference/free layer. (b) Current-induced magnetoresistance oscillations for one representative device for positive current between $0.5 \mu \mathrm{A}$ and $4 \mu \mathrm{A}$ with $0.5 \mu \mathrm{A}$ steps at zero magnetic field. A vertical offset of $2 \mathrm{nW} / \mathrm{GHz}$ is applied to the curves for visual clarity.

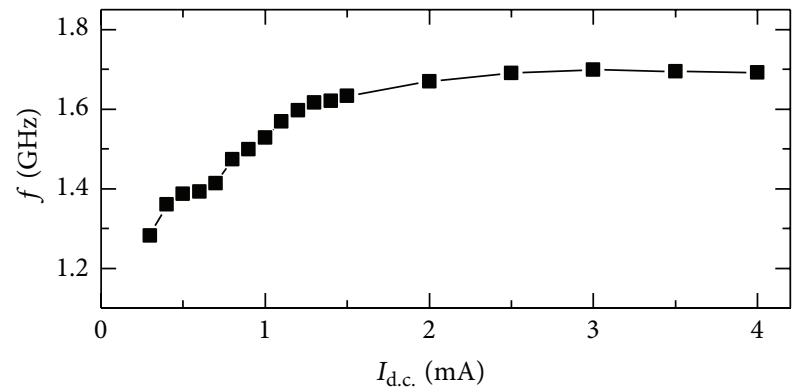

(a)

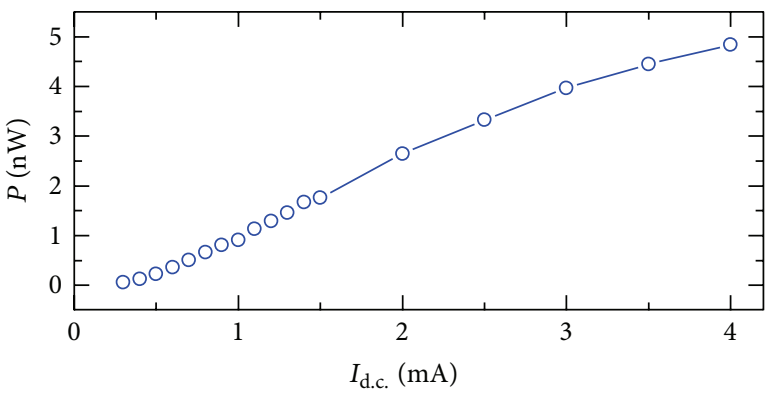

(b)

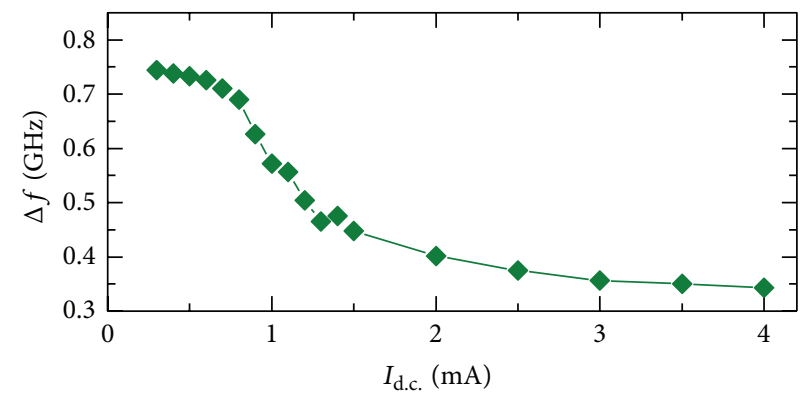

(c)

Figure 4: (a) Frequency, (b) integrated power, and (c) linewidth of the centre mode as a function of applied current.

Finally, we study the dependence of the microwave signal on the external magnetic field $\left(H_{\text {ext }}\right)$. Figure 5 displays the magnetic field dependence of the central mode frequency at $I_{\text {d.c. }}=3 \mathrm{~mA}$. It can be seen that the oscillation frequency can be controlled by adjusting the amplitude of the applied magnetic field. Assuming that the free layer magnetization aligns with applied magnetic field direction in all measurements, the relationship between the frequency $f$ and $H_{\text {ext }}$ is expressed by the Kittel formula [23]:

$$
f=\frac{\gamma}{2 \pi} \sqrt{\left(H_{\mathrm{ext}}+H_{p}\right)\left(H_{\mathrm{ext}}+H_{p}+4 \pi M_{\mathrm{eff}}\right)},
$$

where $\gamma=2.8 \mathrm{MHz} / \mathrm{Oe}$ is the gyromagnetic ratio and $H_{p}$ and $4 \pi M_{\text {eff }}$ are the coupling field and the effective 


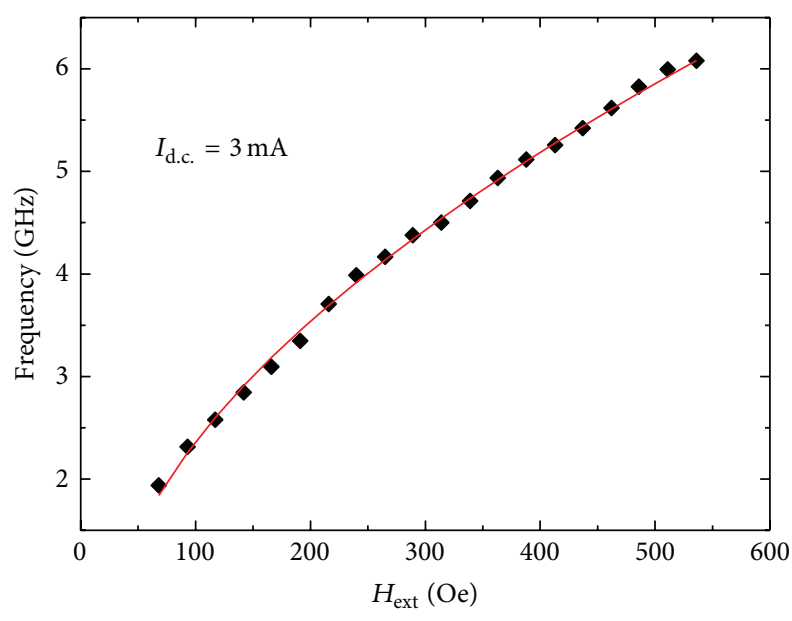

- Exp.

— Fitting

FIgURE 5: The oscillation frequency as a function of applied magnetic field at $I_{\text {d.c. }}=3 \mathrm{~mA}$.

demagnetization field of the free layer. The results are fitted well by this formula as shown in Figure 5 (red line), and $H_{p}$ and $4 \pi M_{\text {eff }}$ are evaluated to be about -20 and 8590 Oe, respectively. The perpendicular anisotropy at the interface of the $\mathrm{CoFeB} / \mathrm{MgO}$ layer results in this reduced $4 \pi M_{\text {eff }}$ value.

\section{Conclusion}

In summary, we have successfully fabricated nanoscale STOs by colloidal lithography and optical lithography instead of the expensive e-beam lithography. The fabricated STO device showed microwave signal generation under d.c. currents. Moreover, the generated microwave signal can be controlled by adjusting the d.c. current or magnetic field. We believe that such a simple and affordable bottom-up approach will drastically speed up the basic research of spin-torque devices and their potential applications.

\section{Conflict of Interests}

The authors declare that there is no conflict of interests regarding the publication of this paper.

\section{Acknowledgments}

The authors would like to thank Nanofabrication Facility and Platform for Characterization and Test of SINANO, Chinese Academy of Sciences. This work was supported by the National Science Foundation of China (11274343, 11474311, 11174341, and 11222432) and the National Key Foundation for Exploring Scientific Instrument of China (2011YQ120053).

\section{References}

[1] T. J. Silva and W. H. Rippard, "Developments in nano-oscillators based upon spin-transfer point-contact devices," Journal of
Magnetism and Magnetic Materials, vol. 320, no. 7, pp. 12601271, 2008.

[2] A. Slavin and V. Tiberkevich, "Nonlinear auto-oscillator theory of microwave generation by spin-polarized current," IEEE Transactions on Magnetics, vol. 45, no. 4, pp. 1875-1918, 2009.

[3] Z. Zeng, G. Finocchio, and H. Jiang, "Spin transfer nano-oscillators," Nanoscale, vol. 5, no. 6, pp. 2219-2231, 2013.

[4] J. C. Slonczewski, "Current-driven excitation of magnetic multilayers," Journal of Magnetism and Magnetic Materials, vol. 159, no. 1-2, pp. L1-L7, 1996.

[5] L. Berger, "Emission of spin waves by a magnetic multilayer traversed by a current," Physical Review B-Condensed Matter and Materials Physics, vol. 54, no. 13, pp. 9353-9358, 1996.

[6] S. I. Klselev, J. C. Sankey, I. N. Krivorotov et al., "Microwave oscillations of a nanomagnet driven by a spin-polarized current," Nature, vol. 425, no. 6956, pp. 380-383, 2003.

[7] W. Rippard, M. Pufall, S. Kaka, S. Russek, and T. Silva, "Directcurrent induced dynamics in $\mathrm{Co}_{90} \mathrm{Fe}_{10} / \mathrm{Ni}_{80} \mathrm{Fe}_{20}$ point contacts," Physical Review Letters, vol. 92, no. 2, Article ID 027201, 4 pages, 2004.

[8] D. Houssameddine, U. Ebels, B. Delaët et al., "Spin-torque oscillator using a perpendicular polarizer and a planar free layer," Nature Materials, vol. 6, no. 6, pp. 441-447, 2007.

[9] A. M. Deac, A. Fukushima, H. Kubota et al., "Bias-driven highpower microwave emission from $\mathrm{MgO}$-based tunnel magnetoresistance devices," Nature Physics, vol. 4, no. 10, pp. 803-809, 2008.

[10] A. Dussaux, B. Georges, J. Grollier et al., "Large microwave generation from current-driven magnetic vortex oscillators in magnetic tunnel junctions," Nature communications, vol. 1, no. 8, 2010.

[11] Z. Zeng, G. Finocchio, B. Zhang et al., "Ultralow-current-density and bias-field-free spin-transfer nano-oscillator," Scientific Reports, vol. 3, article 1426, 2013.

[12] Y. Zhang, H. Zhao, A. Lyle, P. A. Crowell, and J.-P. Wang, "High power and low critical current spin torque oscillation from a magnetic tunnel junction with a built-in hard axis polarizer," Applied Physics Letters, vol. 100, no. 3, Article ID 032405, 2012.

[13] Z. Zeng, P. K. Amiri, I. N. Krivorotov et al., "High-power coherent microwave emission from magnetic tunnel junction nanooscillators with perpendicular anisotropy," ACS Nano, vol. 6, no. 7, pp. 6115-6121, 2012.

[14] S. Kaka, M. R. Pufall, W. H. Rippard, T. J. Silva, S. E. Russek, and J. A. Katine, "Mutual phase-locking of microwave spin torque nano-oscillators," Nature, vol. 437, no. 7057, pp. 389-392, 2005.

[15] A. Ruotolo, V. Cros, B. Georges et al., "Phase-locking of magnetic vortices mediated by antivortices," Nature Nanotechnology, vol. 4, no. 8, pp. 528-532, 2009.

[16] R. Okura, Y. Sakuraba, T. Seki, K. Izumi, M. Mizuguchi, and K. Takanashi, "High-power rf oscillation induced in half-metallic $\mathrm{Co}_{2} \mathrm{MnSi}$ layer by spin-transfer torque," Applied Physics Letters, vol. 99, no. 5, Article ID 052510, 2011.

[17] A. Dussaux, A. V. Khvalkovskiy, P. Bortolotti, J. Grollier, V. Cros, and A. Fert, "Field dependence of spin-transfer-induced vortex dynamics in the nonlinear regime," Physical Review B, vol. 86, no. 1, Article ID 014402, 2012.

[18] H. Fredriksson, Y. Alaverdyan, A. Dmitriev et al., "Hole-mask colloidal lithography," Advanced Materials, vol. 19, no. 23, pp. 4297-4302, 2007.

[19] W. G. Wang, A. Pearse, M. Li et al., "Parallel fabrication of magnetic tunnel junction nanopillars by nanosphere lithography," Scientific Reports, vol. 3, article 1948, 2013. 
[20] S. Ikeda, K. Miura, H. Yamamoto et al., "A perpendicular-anisotropy CoFeB-MgO magnetic tunnel junction," Nature Materials, vol. 9, no. 9, pp. 721-724, 2010.

[21] W. Skowroński, T. Stobiecki, J. Wrona, G. Reiss, and S. van Dijken, "Zero-field spin torque oscillator based on magnetic tunnel junctions with a tilted CoFeB free layer," Applied Physics Express, vol. 5, no. 6, Article ID 063005, 2012.

[22] J.-V. Kim, V. Tiberkevich, and A. N. Slavin, "Generation linewidth of an auto-oscillator with a nonlinear frequency shift: spin-torque nano-oscillator," Physical Review Letters, vol. 100, no. 1, Article ID 017207, 2008.

[23] G. V. Skrotskii and L. V. Kurbatov, Ferromagnetic Resonance, Pergamon Press, 1966. 

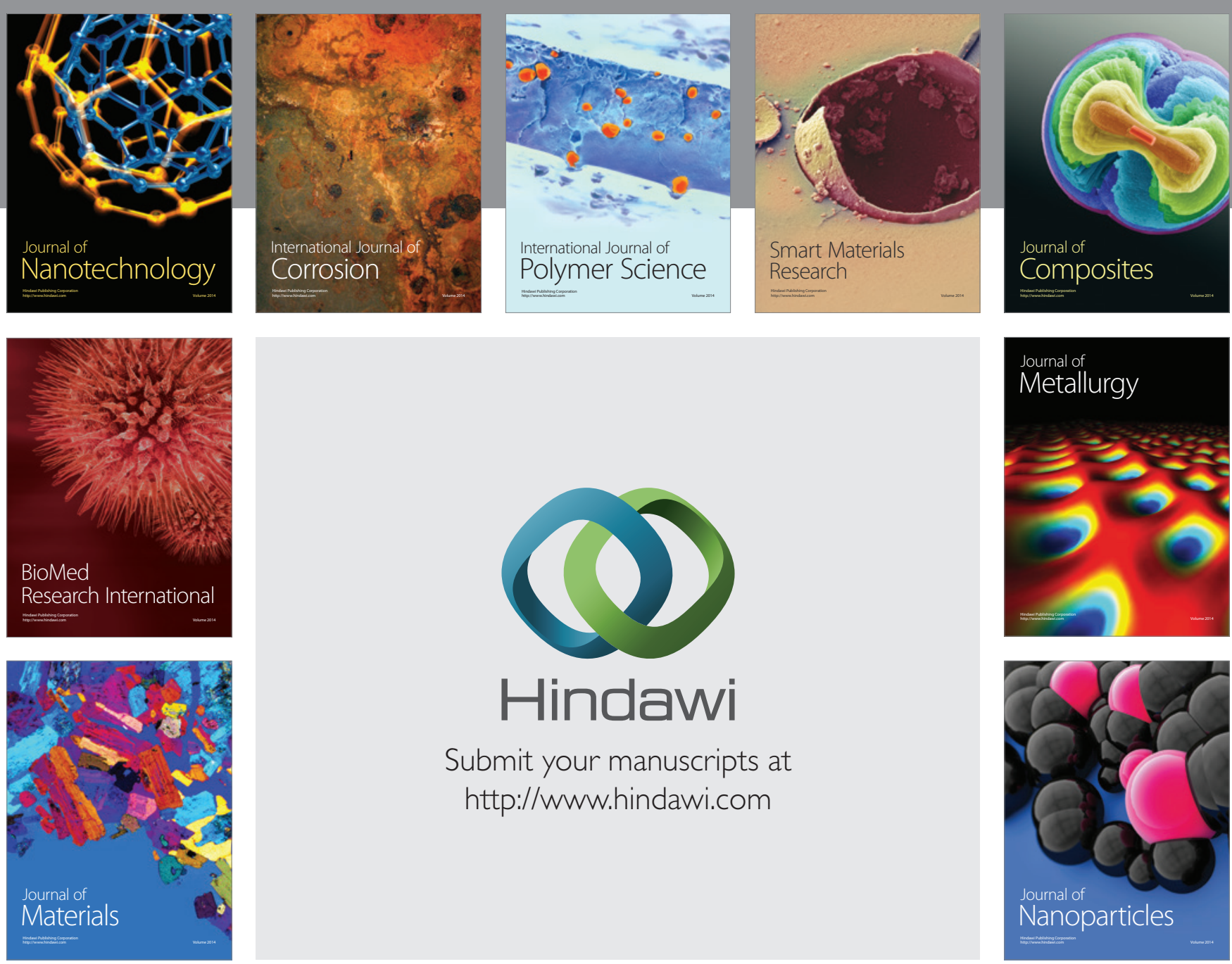

Submit your manuscripts at http://www.hindawi.com
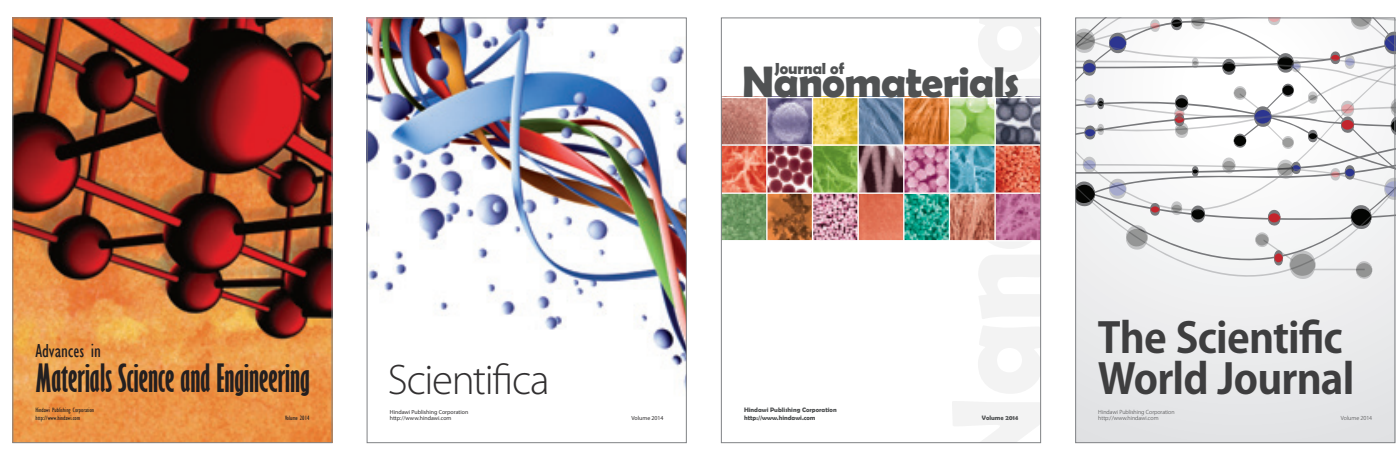

\section{The Scientific World Journal}
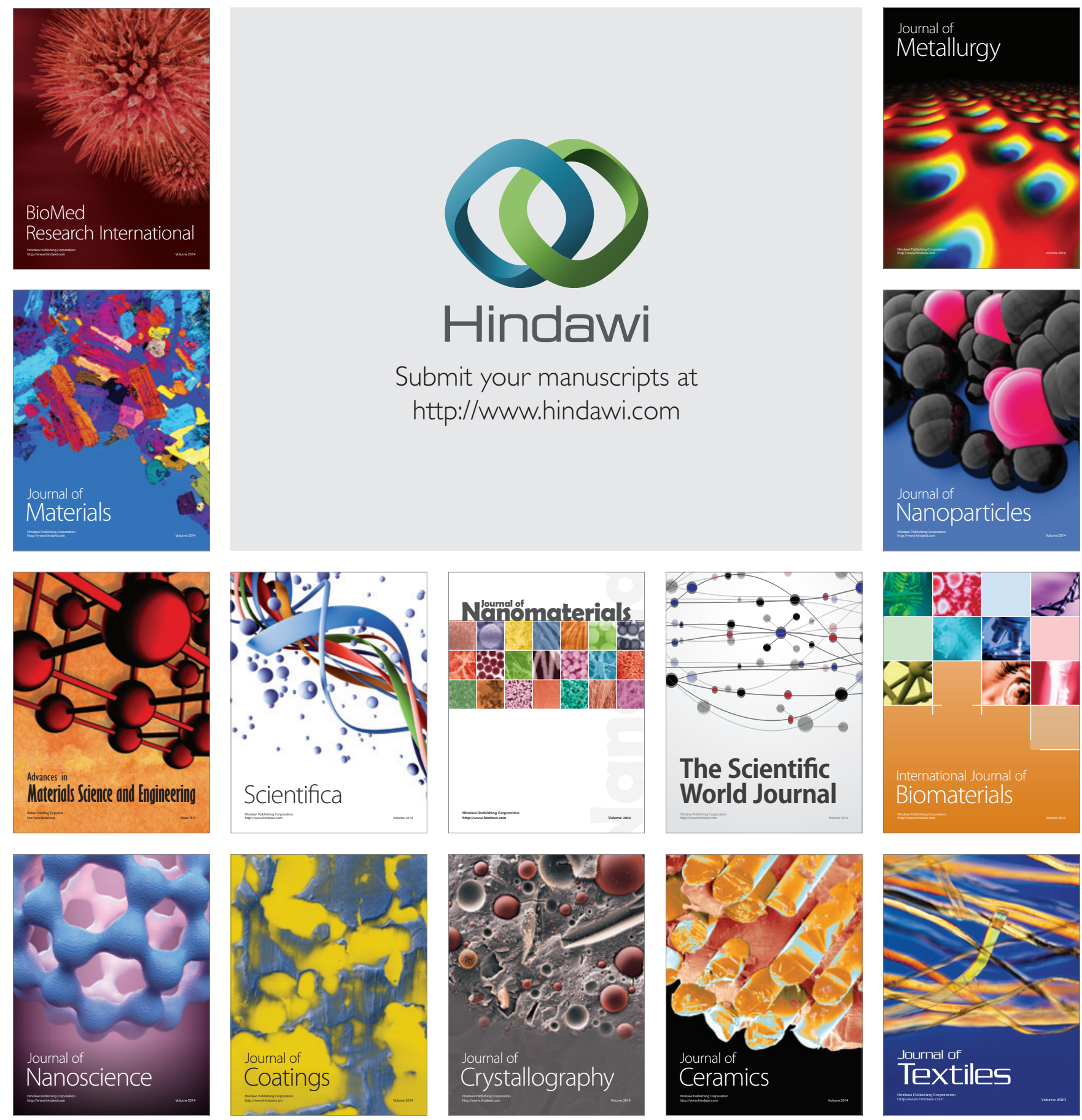